\title{
Teratogens in the human: Current problems
}

\author{
D. F. HAWKINS \\ From the Institute of Obstetrics and Gynaecology, Hammersmith Hospital, London
}

Drugs administered to the mother can affect the fetus by preventing implantation or by causing resorption of the embryo, abortion or intrauterine death. It is a sobering thought that the first of these mechanisms is dignified by the name 'contraception' and that the remainder have far less medicolegal and economic significance than the survival of a defective fetus after a less drastic chemical or physical insult (Law Commission, 1974).

The general principles of teratogenesis in humans have recently been reviewed by Lewis (1974). The majority of drugs are of molecular weight below 1000 and pass the placenta into the fetus. Their rate of transfer is enhanced by small molecular weight, lipophilic properties, and a high concentration of nonionized drug, not bound to plasma protein, in the maternal circulation. Drugs for which detoxication mechanisms, metabolic degradation or urinary excretion are deficient in the fetus are more likely to accumulate.

The extent and nature of a teratogenic effect depend on length of gestation. In general, agents administered before implantation either destroy the embryo or damage cells in the blastocyst which can be replaced by undifferentiated cells which retain totipotency. Maximum susceptibility in man is during organogenesis, from the first week after fertilization until the ninth week, that is, from three to 11 weeks after the last menstrual period. The same stimulus may produce a different anomaly at different stages of gestation, depending on which organs are in a critical stage of development. Some agents show a predilection for causing abnormalities in particular organ systems; sometimes this can be related to a high local tissue concentration or specific influence on a particular metabolic pathway but in other instances the mechanism is unknown. The genetic constitution of the embryo has a major influence on its response, and species differences are the primary hindrance to the screening of agents in animals for teratogenic potential and the interpretation of the relevance of the results to humans. Even in man, racial and familial predisposition to particular anomalies may influence the nature of a teratogenic response.

\section{Recognition of a Teratogenic Effect}

The likelihood that some drugs will cause fetal abnormality can be predicted from knowledge of their mode of action, their pharmacological and toxic actions, and their chemical similarity to known teratogens. Primary testing of most new compounds which may be employed in human therapeutics takes place in animals. Chick embryos and pregnant mice, rats and rabbits are widely used, employing parenteral administration of maximum tolerated doses. Some teratogens will only be detected by the response of a single species; others will cause species specific anomalies. Agents known to be innocuous in man, by virtue of many years of wide therapeutic use in pregnant women, sometimes have a teratogenic effect when given in massive overdose in a selected animal test. On the other hand, whilst the animal tests are likely to detect a major teratogen, this is by no means certain. It is therefore desirable that all drugs intended for widespread use in the pregnant female be tested in primates and then introduced into human therapeutics with extreme caution and close monitoring. There are some situations which are unequivocally life threatening to the mother where a new drug can be reasonably employed, such as a new antibiotic in a serious infection which proves resistant to available medicaments.

The other methods by which adverse effects on fetal development can be detected are epidemiological. When the incidence of a given abnormality is noted to be high or increasing in a community, careful retrospective analysis may reveal the cause. It was by this means that Lenz (1961) found the relationship between phocomelia and thalidomide. Such analyses are not immune from error-the coincident onset of another source of fetal abnormality such as a virus epidemic apparently causing only trivial symptoms can be misleading. In addition it is always possible that it is the disease which the drug is used to treat, or an associated condition, that is the real predisposing factor. Retrospective surveys of the antecedents of a fetal condition of known and consistent incidence are even more susceptible to such errors. They depend on the 
demonstration of correlations which may not have any causal basis. Only when a relevant mode of action of the suspected teratogen in producing the abnormality in susceptible individuals has been demonstrated can the case be regarded as proven as far as those individuals are concerned. Retrospective surveys are nearly always biased by the enhanced recall of mothers who have produced an abnormal baby. Even prospective surveys are open to the problem that it may be the disease and not the drug used to treat it that is the adverse factor. Only many years of extremely large-scale studies of carefully matched and analysed cases will serve to determine the true magnitude of the effect of drug therapy during pregnancy in relation to congenital abnormality.

\section{Major Teratogens in Man}

These include thalidomide which characteristically causes phocomelia; actinomycin, folic acid antimetabolites such as methotrexate, and alkylating antimitotic drugs, which cause fetal death or a variety of anomalies if the fetus survives; tetracycline which discolours and causes enamel hypoplasia in deciduous teeth; and radioactive ${ }^{131}$ I which causes fetal goitre. There are very few circumstances in which there is no alternative to the use of these agents in pregnancy. If any of these agents, except tetracycline, is vital to the survival of the mother then the pregnancy should be terminated. Similar considerations apply to the use of irradiation of the pelvis or whole body to treat cancer or related conditions and to open-heart surgery with the use of a bypass circulation which will cause prolonged fetal anoxia. In the last case, it must be remembered that the teratogenic effects of anoxia in early pregnancy, should the fetus survive, are well established experimentally.
Therapeutic Agents which may Convey a Small Risk to the Fetus

A number of drugs used in pregnancy in this class are shown in table $\mathrm{I}$.

When a patient for whom treatment with one of these agents is indicated undertakes a pregnancy, what is said to her and her attitude are of great importance. Any pregnant woman, on drug therapy or not, told of the calculated risk that her baby will be abnormal will react adversely. Such a statement shows lack of perspective. If odds are required, then the patient wishes to know the chance that her baby will be normal. In conditions requiring treatment with the drugs indicated in table $I$, the patient is nearly always aware that there is some risk that her pregnancy may be unsuccessful. In general the major hazard is the maternal disease itself, the chances of drug teratogenicity being small in comparison. Both hazards are much reduced by careful clinical management and close control of drug treatment.

For example, the patient who has had recurrent pulmonary emboli in previous pregnancies may need anticoagulant therapy throughout a pregnancy. With subcutaneous heparin in the first trimester, then a well controlled warfarin regimen until 38 weeks, an intravenous heparin infusion for a few days, discontinued for elective induction of labour, careful management of the third stage of labour, and heparin and then warfarin in the puerperium, the risk to the fetus should be minimal. Similarly the thyrotoxic patient carefully managed with a combination of partial thyroidectomy and then, if necessary, the small amounts of antithyroid drugs required to achieve a euthyroid status, should pose only a tiny risk to the fetal thyroid. Close control of therapy in maternal disorders such as essential hypertension, diabetes mellitus and conditions requiring
Antithyroid drugs; iodides

Azathioprine; mercaptopurine

Barbiturates (large doses)

Chloroquine

Coumarin anticoagulants

Diazepam and related benzodiazepines (large doses)

Frusemide

Ganglion blockers

Glucocorticoids

Live vaccines

Narcotics

19-Nor-steroids

Phenytoin, primidone

Rauwolfia alkaloids

Streptomycin, kanamycin, gentamicin, vancomycin
Fetal goitre

Susceptibility to virus infection

Newborn withdrawal syndrome

Retinal damage; multiple abnormalities

Fetal haemorrhage; rarely, fetal abnormalities

Newborn withdrawal syndrome

Maternal intravascular dehydration and reduced placental perfusion leading to fetal growth retardation

Paralytic ileus

Susceptibility to virus infection; very rarely cleft palate

Fetal virus infection

Respiratory depression; newborn withdrawal syndrome

Virilisation of female fetus (transient clitoral hypertrophy; rarely labial fusion)

Fetal abnormalities, fetal bleeding; newborn withdrawal syndrome

Newborn depression syndrome

Sometimes minor 8 th nerve damage detected on audiometry, very rarely deafness

Skeletal abnormalities

Table I Some therapeutic agents believed to convey a teratogenic risk in a small proportion of cases 
steroid therapy in pregnancy should accomplish a satisfactory outcome to both mother and fetus.

\section{Suspicions of Teratogenic Activity}

Since the thalidomide disaster, many reports have appeared in the medical literature based on suspicion alone (table II). All the fallacies have been incorporated -extrapolation from animal data, selected populations, assumptions that a correlation means a causal relationship, retrospective surveys and unsubstantiated theory. Whilst it is highly desirable that such suspicions should be aired at the earliest opportunity, there are dangers when they are reiterated as established fact. It has been said that far more babies were damaged or lost in the years following the first reports on the risk of the use of radiographs in pregnancy from failure to radiograph than were ever at risk for leukaemia even if that risk was as at first thought. Withdrawal of therapy from a pregnant epileptic is more likely to cause fetal damage than its continuation with appropriate precautions. The consequences of failure to treat even minor conditions such as hyperemesis or trichomoniasis could outweigh the potential hazards of therapy.

\section{Current Problems}

\section{SEX HORMONES}

It has recently been suggested that the use of oral contraceptives can predispose to congenital abnormality. The work is based on retrospective surveys, with all their difficulties. The first suspicion is an association with limb reduction defects. This is a relatively rare malformation whose incidence in New York State $(0.25$ per 1000 births in 1973) has been rising in parallel with increased use of oral contraceptives. Janerich et al (1974) found an increased proportion of mothers of such babies had discontinued oral contraceptives in the cycle immediately before conception, compared with control series. It is possible that this might be linked with the 'aging ovum' situation. Ovulation is often delayed in the first cycle and conception late in the cycle may be linked with congenital abnormalities (Cross, 1961; Iffy, 1963; Hertig, 1967). Also, it is not surprising that neonatal jaundice has been reported to be associated with previous maternal use of oral contraceptives. The ease with which the maturity of a pregnancy may be underestimated and consequently labour inadvertently induced prematurely may be the factor responsible. The association has not been confirmed in subsequent better controlled studies (Gould et al, 1974).

In addition, Janerich et al (1973) reported that six of their 108 mothers of babies with limb reduction defects had become pregnant whilst taking oral contraceptives, and six more had been given sex steroids during the pregnancy. It was a surprising and perhaps significant finding that in every one of the mothers who had had oral hormones in early pregnancy, the sex of the affected baby was male. Some support for the concept that sex hormone administration in early pregnancy may convey a small teratogenic risk has come from Colorado, where Nora and Nora (1975) have described a syndrome of multiple abnormalities-vertebral, anal, cardiac, tracheo-oesophageal, renal and limb deformities, which they feel may be associated. In England and Wales, Greenberg et al (1975) have suggested that an increased proportion of mothers of abnormal babies have been given doses of hormones in early pregnancy in the form of 'oral pregnancy tests' to see if withdrawal bleeding occurs.

It has been known for years (Wilkins, 1960) that 19-nor-progestagens given in early pregnancy can give rise to a very minor degree of virilization in female fetuses. When this occurs it is usually just clitoral hypertrophy which resolves over the first year of life, but occasionally fusion of the labioscrotal folds requires division. More serious are the cluster of cases of adenocarcinoma of the vagina in teenage girls whose mothers had been given stilboestrol during pregnancy in the Boston area (Herbst et al, 1971). On the other hand, Kinlen et al (1974) were unable to pinpoint a single case amongst the 12000 patients they estimated had been given stilboestrol in Great Britain.

The number of cases of fetal abnormality that could possibly be attributed to sex hormones is small and, in most instances, the case is unproven. Oral contraceptive failure or administration of an oral pregnancy test do not qualify as a 'substantial risk' under the Abortion Act. With the oral contraceptives the dose of hormones has now been reduced to a very low level. On the other hand there is no valid indication for using oral pregnancy tests, and there is no evidence that either stilboestrol or 19-norsteroids have any value in pregnancy maintenance (Hawkins, 1974) and their use should be abandoned. Only with a woman suffering from primary habitual abortion who has demonstrable progesterone deficiency is there any case at all for hormone supplements, and 17-hydroxyprogesterone, which has not been indicted, is the most appropriate agent.

\section{Antiepileptic Treatment}

It is now clear that phenytoin and related agents can cause folic acid deficiency in pregnancy and, probably in consequence, give rise to congenital ab- 
normalities in a small proportion of babies. In addition, there is a risk of fetal bleeding during the confinement and the newborn babies are susceptible to a sedative withdrawal syndrome. It is important that epileptic women should plan their pregnancies and should start taking folic acid supplements before they even conceive and continue throughout the pregnancy. This is in order that any necessary adjustment can be made to the dose of phenytoin and that adequate folic acid is available during implantation and organogenesis. Parenteral vitamin $\mathrm{K}$ should be given to the mother early in labour and the newborn closely supervised in a neonatal unit for three days after delivery.

With epileptics controlled on barbiturates, the main risk is that of sedative withdrawal in the newborn, which requires careful management (Desmond et al, 1972).

\section{Sulphonamides}

Experimentally, only sulphafurazole, long-acting sulphonamides and trimethoprim with sulphamethoxazole have raised any suspicion of embryopathic activity. In practice there is no evidence of teratogenicity in man, but as there are alternative sulphonamides, the first two are possibly best avoided, and if trimethoprim with sulphamethoxazole is used in pregnancy folic acid supplements should be given.

In the newborn sulphonamides can compete for binding sites for bilirubin conjugation and increase plasma bilirubin levels. It has been suggested (Stirrat and Beard, 1973) that the drugs are contraindicated in pregnancy in that they could cause neonata, jaundice and kernicterus in the newborn. In factl although the drugs have been very widely used in pregnancy for many years, there is no evidence that this phenomenon ever occurs with short-acting sulphonamides. Kernicterus is extremely rare in Great Britain, except in association with rhesus isoimmunization, and no cases connected with sulphonamide therapy in pregnancy have been reported to the Committee on Safety of Medicines. Oral sulphonamides are still the first line of attack for urinary infections in pregnancy; they are of no use in labour due to poor absorption.

\section{Metronidazole}

Following a study by Rustia and Shubik(1972), which suggested that mice fed on large doses of metronidazole throughout their lives had an increased incidence of adenomata in the lung, and work by Voogd et al (1974) showing that the drug increased the mutation rate in certain bacteria, efforts were made to ban the use of metronidazole entirely in the United States of America. Its use to treat trichomoniasis in pregnancy has been criticized. Further animal studies have failed to confirm that there is any carcinogenic or teratogenic risk associated with metronidazole. In fact, over
Alcohol

Amine oxidase inhibitors

Amphetamines

Antacids in first trimester

Antiemetics, cyclizine

Aspirin; phenacetin

Barbiturates in ordinary doses

Cannabis smoking

Chlorpromazine (large doses)

Chlorthiazide

Idoxuridine

Iron preparations in first trimester

Lithium carbonate

Lysergic acid diethylamide

Meprobamate in first trimester

Metronidazole

Methyldopa (large doses)

Oral contraceptives

Oral pregnancy tests

Oral hypoglycaemic agents

Podophyllin

Propranolol

Quinine

Stilboestrol

Sulphonamides

Tobacco smoking

Tricyclic antidepressants

$\mathrm{X}$-ray exposure
Intrauterine and infant growth retardation; fetal abnormalities Fetal abnormalities; perinatal death

Fetal thrombocytopenia

Fetal abnormalities; fetal bleeding

Fetal abnormalities; minor skin malformations

Retinal damage

Fetal thrombocytopenia

Table II Some drugs on which suspicion has been cast ${ }^{1}$

'Where no risk is indicated, an association with an overall increase in fetal abnormality has been suspected.

Meconium ileus

Limb and gastro-intestinal abnormalities; neonatal jaundice

Limb and other abnormalities, particularly in male fetuses

Fetal abnormalities; fetal hypoglycaemia and hypothyroidism

Intrauterine growth retardation

Fetal abnormalities; fetal thrombocytopenia; middle ear damage

Vaginal adenosis and adenocarcinoma

Fetal abnormalities; neonatal jaundice

Prematurity, intrauterine growth retardation; increased perinatal mortality

Fetal abnormalities; perinatal death

Fetal abnormalities; infant leukaemia and malignant disease 
6500000 women have been treated with metronidazole and there has never been any suggestion of carcinogenicity. Over 2500 cases of women treated during pregnancy are reported in the medical literature and no atypical incidence of congenital abnormalities has been recorded. The major retrospective surveys have failed to suggest any teratogenic association. It must also be taken into account that vaginal trichomoniasis is commonly associated with in-situ and invasive carcinoma of the cervix, and that women with untreated trichomonal infections have a tendency to go into premature labour of the type that starts with rupture of the membranes.

The evidence on metronidazole should be considered against the background that when the drug was introduced as an oral agent in Great Britain 15 years ago, its efficacy was rapidly recognized and it swept the market of all the relatively ineffective local trichomonacides. The current criticisms have arisen in the United States of America within a year or two of the introduction of oral metronidazole to the American market.

In practice, avoidance of the use of oral metronidazole in pregnancy during the first 16 weeks should relieve any doubts about its potential, though the case is entirely unproven.

\section{Barbiturates}

Obstetricians have a justifiable scepticism about criticism of the use of barbiturates. The drugs have been enormously widely used during pregnancy for over 40 years, with much benefit to the patients. Fifteen years ago suspicions were voiced that the drugs caused liver damage in the newborn. Seven years ago we were advised that they stimulated formation of detoxifying enzymes in the fetal liver which tended to protect the baby against birth asphyxia and neonatal jaundice. Most recently (see British Medical Journal, 1972) we have been told of the possiblity of generating behavioural abnormalities in the babies. Although barbiturates are used so widely in pregnancy, the retrospective surveys are equivocal with regard to teratogenesis. The slightly increased incidence of abnormalities found by Nelson and Forfar (1971) may well be accounted for by the complications of pregnancy for which barbiturates were prescribed. Detailed analysis of the data obtained in the Royal College of General Practitioners' survey (1975) showed no suggestion of a cause-and-effect relationship between barbiturate therapy and congenital malformations.

It seems reasonable to continue to use barbiturates in pregnancy in conditions where they are indicated, and perhaps preferable to prescribing newer drugs about which less is known. The babies of patients given moderate or large doses of barbiturates-or, for that matter, diazepam-up to the confinement, should be observed for any signs of a sedative withdrawal syndrome (Desmond et al, 1972; Cree et al, 1973).

\section{Tranquillizers}

A number of antihistamines and phenothiazines used in the treatment of morning sickness have been successively indicted and cleared of suspicion. The widely used drugs, promethazine and its theophylline conjugate, have not been seriously questioned. Cyclizine and diphenhydramine are still under review and should probably be avoided.

Though there is no clear evidence with respect to monamine oxidase inhibitors and tricyclic antidepressants, obstetricians do not like them and anaesthetists do not like them. The suspicion is there that the baby which has been subjected to them in the intrauterine environment is at risk, and most practitioners adopt a reactionary attitude when prescribing sedatives and tranquillizers for pregnant women.

Lysergic acid diethylamide can be teratogenic in experimental circumstances. Isolated cases of congenital abnormalities have been seen in women who take drugs during pregnancy. It is difficult to obtain reliable histories and these cases could well be due to other teratogenic factors. There is as yet no significant evidence with respect to the effects of cannabis smoking on the fetus.

\section{Alcohol}

Attention has recently been drawn to a syndrome in the newborn associated with alcoholism in pregnancy (Jones et al, 1973; Jones and Smith, 1973). Microcephaly, skull malformations and other anomalies can occur. More consistent are intrauterine growth retardation, infant growth retardation and delayed behavioural development. The babies of alcoholic mothers need careful observation and follow up.

\section{Tobacco Smoking}

Since the first demonstration by Simpson (1957) of a correlation between smoking in pregnancy and retarded intrauterine growth, a mass of evidence on this correlation has built up (U.S. Department of Health, Education and Welfare, 1973). Comments made at the time (Hawkins, 1959) remain unanswered - no causal mechanism has yet been clearly demonstrated. It is quite clear that smoking in pregnancy is associated with small-for-dates babies, and there may be an association with premature labour and 
perhaps with an increased perinatal mortality, though the last has not been confirmed in large-scale American studies. What is often said, but is quite unproven, is that persuading women to stop smoking in pregnancy improves the prognosis. The work of Butler et al (1972), who showed that women who reported ceasing to smoke after the fourth month had a normal prognosis for the baby, is really unhelpful. It is a retrospective survey, susceptible to biased recall. In addition, women who stop smoking in pregnancy of their own volition are a special group who are exhibiting a pica. Women who are persuaded to stop smoking in pregnancy are a totally different group. In a recently controlled prospective study conducted in three London teaching hospitals the usual association was confirmed but it was shown that those women who were persuaded to stop smoking did not achieve an improved prognosis (Donovan, J., personal communication, 1975).

The situation therefore harks back to the original contention that there are a proportion of women who have in common the attributes that they smoke and that they produce small babies!

\section{Radiographs and Ultrasound}

The recent publicity given to the desirability of avoiding all but urgent radiological examinations during the second half of the menstrual cycle (Royal College of Radiologists, 1975) to avoid any risk to an early conceptus is obviously reasonable. Similarly the avoidance of non-essential radiographs in pregnancy, the precautions taken to minimize exposure and the avoidance of examinations involving multiple exposures are sound practice. On the other hand it must be recalled that the numerical risk to the fetus is extremely small. In these circumstances the fact that a woman has inadvertently had a radiological examination in early pregnancy cannot be taken to constitute the substantial risk of physical or mental abnormality in the baby which constitutes grounds for terminating the pregnancy under the Abortion Act.

Much play has been made in recent years of the idea that many radiological examinations in pregnancy could be replaced by the use of ultrasound. It should be borne in mind that whether or not ultrasound examinations can have teratogenic long-term effects on the fetus is quite unknown.

\section{Conclusion}

The practitioner who restricts his prescribing for pregnant women to circumstances where medication is really indicated, and employs medicines which have been widely used in pregnancy for many years without apparent harm is unlikely to contribute significantly to the incidence of congenital malformations.

\section{References}

British Medical Journal (1972). Neonatal behaviour and maternal barbiturates. (Leading article). Brit. med. J., 4, 63-64.

Butler, N. R., Goldstein, H., and Ross, E. M. (1972) Cigarette smoking in pregnancy: its influence on birth weight and perinatal mortality. Brit. med. J., 2, 127-130.

Cree, J. E., Meyer, J., and Hailey, D. M. (1973). Diazepam in labour: its metabolism and effect on clinical condition and thermogenesis of newborn. Brit. med. J., 4, 251-255.

Cross, R. G. (1961). Prevention of anencephaly and foetal abnormalities by a preconceptional regimen. Lancet, 2 , 1124.

Desmond, M. M., Schwanecke, R. P., Wilson, G. S., Yasunaga, S., and Burgdorff, I. (1972). Maternal barbiturate utilization and neonatal withdrawal symptomatology. J. Pediat., 80, 190-197.

Gould, S. R., Mountrose, U., Brown, D. J., Whitehouse, W. L., and Barnardo, D. E. (1974). Influence of previous oral contraception and maternal oxytocin infusion on neonatal jaundice. Brit. med. J., 3, 228-230.

Greenberg, G., Inman, W. H W, Weatherall, J. A. C., and Adelstein, A. M. (1975). Hormonal pregnancy tests and congenital malformations. (Letter). Brit. med. J., 2, 191192.

Hawkins, D. F. (1959). Birth weight and maternal smoking. (Letter). Brit. med. J., 2, 820.

Hawkins, D. F. (1974). Sex hormones in pregnancy. In Obstetric Therapeutics, edited by D. F. Hawkins, ch. 3, pp. 106-150. Baillière Tindall, London.

Herbst, A. L., Ulfelder, H., and Poskanzer, D. C. (1971). Adenocarcinoma of the vagina: association of stilbestrol therapy with tumor appearance in young women. New Engl. J. Med., 284, 878-881.

Hertig, A. T. (1967). Human trophoblast: normal and abnormal. Amer. J. clin. Path., 47, 249-268.

Iffy, L. (1963). The time of conception in pathological gestations. Proc. roy. Soc. Med., 56, 1098-1100.

Janerich, D. T., Piper, J. M., and Glebatis, D. M. (1974). Oral contraceptives and congenital limb-reduction defects. New Engl. J. Med., 291, 697-700.

Jones, K. L., and Smith, D. W. (1973). Recognition of the fetal alcohol syndrome in early infancy. Lancet, 2, 999. 1001.

Jones, K. L., Smith, D. W., Ulleland, C. N., and Streissguth, A. P. (1973). Pattern of malformation in offspring of chronic alcoholic mothers. Lancet, 1, 1267-1271.

Kinlen, L. J., Badaracco, M. A., Moffett, J., and Vessey, M. P. (1974). A survey of the use of oestrogens during pregnancy in the United Kingdom and of the genitourinary cancer mortality and incidence rates in young people in England and Wales. J. Obstet. Gynaec. Brit. Cwlth, 81, 849-855.

Law Commission (1974). Report on Injuries to Unborn Children. Report No. 60. HMSO, London.

Lenz, W. (1961). Kindliche Missbildungen nach Medikament-Einnahme währand der Gravidität? Dtsch. med. Wschr., 86, 2555.

Lewis, B. V. (1974). The action of drugs on the uteroplacental circulation and the developing fetus. In Obstetric Therapeutics, edited by D. F. Hawkins, ch. 2, pp. 71-105. Baillière Tindall, London.

Nelson, M. M., and Forfar, J. O. (1971). Associations be- 
tween drugs administered during pregnancy and congenital abnormalities of the fetus. Brit. med. J., 1, 523-527.

Nora, A. H., and Nora, J. J. (1975). A syndrome of multiple congenital anomalies associated with teratogenic exposure. Arch. environ. Hlth, 30, 17-21.

Royal College of General Practitioners, Birmingham Research Unit (1975). Morbidity and drugs in pregnancy. J. roy. Coll. gen. Pract., 25, 631-645.

Royal College of Radiologists (1975). Implementation of "The 10 Day Rule". Royal College of Radiologists, London.

Rustia, M., and Shubik, P. (1972). Induction of lung tumors and malignant lymphomas in mice by metronidazole. $J$. nat. Cancer Inst., 48, 721-729.

Simpson, W. J. (1957). A preliminary report on cigarette smoking and the incidence of prematurity. Amer. $J$ Obstet. Gynec., 73, 808-815.

Stirrat, G. M., and Beard, R. W. (1973). Drugs to be avoided or given with caution in the second and third trimesters of pregnancy. Prescribers' J., 13, 135-140.

U.S. Department of Health, Education and Welfare (1973). Pregnancy. In The Health Consequences of Smoking, ch. 4, pp. 103-149. Washington, D.C.

Voogd, C. E., Van der Stel, J. J., and Jacobs, J. A. (1974). The mutagenic action of nitroimidazoles. I. Metronidazole, nimorazole, dimetridazole and ronidazole. Mutat. Res., 26, 483-490.

Wilkins, L. (1960). Masculinization of female fetus due to use of orally given progestins. $J$ Amer. med. Ass., 172, 10281032. 